\title{
UNIVALENT FUNCTIONS WITH UNIVALENT DERIVATIVES
}

BY S. M. SHAH ${ }^{1}$ AND S. Y. TRIMBLE

Communicated by R. Creighton Buck, August 8, 1968

1. Introduction. Let $S$ denote as usual the family of normalized functions

$$
f(z)=z+a_{2} z^{2}+\cdots a_{n} z^{n}+\cdots,
$$

which are holomorphic and univalent in the unit disc $D$. We consider functions $f(\in S)$ such that most derivatives $f^{(k)}$ are univalent in $D$. Our conclusion is that $f$ must be entire.

Let $E$ denote those functions $f(\in S)$ which have the property that for each $n \geqq 1, f^{(n)}$ is univalent in $D$. Let

$$
\alpha=\sup \left\{\left|a_{2}\right| ; f \in E\right\} .
$$

2. Main results. The first theorem shows that if $f \in E$, then $f$ must be an entire function of exponential type. Similar results under somewhat weaker hypotheses are possible. These are contained in the second theorem, the proof of which will appear elsewhere.

THEOREM 1. If $f \in E$, then for $n \geqq 2$,

$$
\left|a_{n}\right| \leqq(2 \alpha)^{n-1} / n !
$$

and

$$
\pi / 2 \leqq \alpha<1.7208 .
$$

Furthermore, $f$ is an entire function of exponential type such that for every $r$, we have

$$
M(r, f) \leqq\{\exp (2 \alpha r)-1\} / 2 \alpha .
$$

Proof. Since $f^{(n)}$ is univalent in $D, a_{n+1} \neq 0$. Define $F_{n}$ in $D$ by

$$
F_{n}(z)=\frac{f^{(n)}(z)-n ! a_{n}}{(n+1) ! a_{n+1}} .
$$

Then $F_{n} \in E$, and

\footnotetext{
1 The research of this author is supported by the National Science Foundation under Grant GP-7544.
} 


$$
F_{n}(z)=z+\frac{(n+2) a_{n+2}}{2 a_{n+1}} z^{2}+\cdots .
$$

It follows from (1.2) that

$$
\left|a_{n+2}\right| \leqq \frac{2 \alpha\left|a_{n+1}\right|}{n+2} .
$$

An inductive argument then establishes (2.1). The fact that $f$ is entire follows from (1.1) and (2.1). Further, we have

$$
\begin{aligned}
|f(z)| & =\left|z+\sum_{2}^{\infty} a_{n} z^{n}\right| \\
& \leqq \sum_{n=1}^{\infty} \frac{(2 \alpha)^{n-1}}{n !}|z|^{n} \\
& =\{\exp (2 \alpha|z|)-1\} / 2 \alpha .
\end{aligned}
$$

In particular, if $z \in D$, then

$$
|f(z)|<\{\exp (2 \alpha)-1\} / 2 \alpha .
$$

It is known [2, p. 223] that if $f \in E, \lambda \geqq 1$, and $|f(z)|<M$ for $z \in D$, then $\left|a_{3}-\lambda a_{2}^{2}\right| \leqq(4 \lambda-5) M^{-2}-8(\lambda-1) M^{-1}+4 \lambda-3$. Letting $\lambda=1$, this becomes $\left|a_{2}^{2}-a_{3}\right| \leqq 1-M^{-2}$. Using (2.1) and (2.4), we have

$$
\left|a_{2}\right|^{2} \leqq 1-4 \alpha^{2} /\left(e^{2 \alpha}-1\right)^{2}+2 \alpha^{2} / 3 .
$$

The right-hand expression in (2.5) is independent of $f$. It follows from (1.2) that

$$
\alpha^{2} \leqq 3\left(1-4 \alpha^{2} /\left(e^{2 \alpha}-1\right)^{2}\right) .
$$

This implies that $\alpha<1.7208$.

The lower bound in (2.2) is the second coefficient of the function

$$
\phi(z)=\frac{1}{\pi}\left(e^{\pi z}-1\right) .
$$

Since $\phi \in E,(2.2)$ is established.

REMARKS. (i) It is easily seen that $E$ is a normal compact family in $D$, and so there must exist in $E$ a function whose second coefficient is $\alpha$.

(ii) The converse of this theorem is false. For example, let $\Psi(z)$ $=\sin z$. Then $\Psi \in S$ and (2.1)-(2.4) are satisfied. But $\Psi$ is not in $E$.

(iii) Suppose $f(z)=z+a_{2} z^{2}+\cdots$ is regular and mean 1-valent in $D$ and that all derivatives $f^{(k)}$ are also mean 1-valent in $D$. Then 
$\left|a_{2}\right| \leqq 2[1$, p. 99] and our argument shows that $f$ is an entire function of exponential type.

THEOREM 2. Let $f(z)$ be holomorphic in $|z|<R$.

$$
f(z)=\sum_{k=0}^{\infty} a_{k} z^{k}, \quad|z|<R(R>0) .
$$

(i) Let $\left\{n_{p}\right\}_{p=1}^{\infty}$ be an increasing sequence of positive integers such that for each $p, f^{\left(n_{p}\right)}$ is univalent in $D$. Then

$$
\liminf _{p \rightarrow \infty}\left(n_{1} n_{2} \cdots n_{p}\right)^{1 / n_{p}} \leqq R .
$$

In particular, if $n_{p+1}-n_{p}=o\left(\log n_{p}\right)$ then $f$ is an entire function.

(ii) Let $\left\{\rho_{n}\right\}_{n=0}^{\infty}$ be a sequence of positive numbers such that for each $n, f^{(n)}$ is univalent in $|z|<\rho_{n}$. Then

$$
\liminf _{n \rightarrow \infty}\left(n\left(\rho_{0} \rho_{1} \cdots \rho_{n}\right)^{1 / n}\right) \leqq 4 e R .
$$

In particular, if

$$
\lim _{n \rightarrow \infty}\left(n\left(\rho_{0} \rho_{1} \cdots \rho_{n}\right)^{1 / n}\right)=\infty,
$$

then $f$ is entire.

3. Functions in $E$. Every function $f \in E$ must be of the form

$$
f(z)=z e^{\beta z} \prod_{1}^{\infty}\left(1+\frac{z}{a_{n}}\right) \exp \left(-\frac{z}{a_{n}}\right)
$$

where or of the form

$$
|\beta| \leqq \alpha, n(r)=O(r),\left|a_{1}\right|>1 \text { and } \sum_{\left|a_{n}\right| \leq r} \frac{1}{a_{n}}=O(1) ;
$$

$$
f(z)=z e^{\beta z} \prod_{1}^{\infty}\left(1+\frac{z}{a_{n}}\right)
$$

where

$$
\sum 1 /\left|a_{n}\right|<\infty, \quad\left|a_{1}\right|>1, \quad\left|\beta+\sum_{1}^{\infty} \frac{1}{a_{n}}\right| \leqq \alpha .
$$

Obviously these conditions are not sufficient for $f$ to be in $E$.

THEOREM 3. Let $\left\{a_{n}\right\}_{n=1}^{\infty}$ be a sequence of complex numbers such that $1 \leqq\left|a_{1}\right|<\left|a_{2}\right|<\cdots, \arg a_{1}=\arg a_{2}=\cdots=\gamma$ (say) and $\sum 1 /\left|a_{n}\right|$ $<\infty$. Let $\arg \beta=-\gamma$ if $\beta \neq 0$ and let 


$$
f(z)=e^{\beta z} \prod_{1}^{\infty}\left(1+\frac{z}{a_{n}}\right), F(z)=z f(z), G(z)=(f(z)-1) / f^{(1)}(0) .
$$

Denote by $\left\{-b_{n}\right\}_{1}^{\infty}$ the zeros of $f^{\prime}(z)$ and by $\left\{-B_{n}\right\}_{1}^{\infty}$ the zeros of $F^{\prime}(z)$.

(i) If

$$
|\beta|+\sum_{1}^{\infty} 1 /\left(\left|b_{n}\right|-1\right) \leqq 1,
$$

then $f, f^{\prime}, f^{\prime \prime}, \cdots$ map $D$ univalently onto convex domains and $G \in E$.

(ii) If $\left|b_{1}\right| \geqq 1$ and

$$
|\beta|+\sum_{2}^{\infty} 1 /\left(\left|b_{n}\right|-1\right) \leqq 1,
$$

then $f, f^{\prime}, f^{\prime \prime}, \cdots$ are all close-to-convex on $D$ and $G \in E$.

(iii) If

$$
\left(\sum_{1}^{\infty} 1 /\left|b_{n}\right|\right)+|\beta| \leqq \log 2
$$

then $G \in E$.

(iv) If

$$
|\beta|+\sum_{1}^{\infty} 1 /\left(\left|B_{n}\right|-1\right) \leqq 1,
$$

then $F, F^{\prime}, \ldots$ map $D$ univalently onto convex domains and $F \in E$.

(v) If $\left|B_{1}\right| \geqq 1$ and

$$
|\beta|+\sum_{2}^{\infty} 1 /\left(\left|B_{n}\right|-1\right) \leqq 1,
$$

then $F, F^{\prime}, \cdots$ are all close-to-convex on $D$ and $F \in E$.

(vi) If

$$
|\beta|+\sum_{1}^{\infty} 1 /\left|a_{n}\right| \leqq \frac{\log 2}{2},
$$

then $F \in E$.

The proof of this theorem will appear elsewhere. Note that the conditions for (i) are satisfied if $\beta=0,\left|a_{1}\right| \geqq 2,\left|a_{n+1}\right| \geqq 2^{n}\left|a_{n}\right|$ $(n=1,2, \cdots)$. The conditions for (ii) are satisfied if $\beta=0,\left|a_{1}\right| \geqq 1$, $\left|a_{n+1}\right| \geqq(1.525)^{n}\left|a_{n}\right|(n=1,2, \cdots)$. Note also that the function $\phi$ in Theorem 1 is of the form (3.1). (See also [3], [4].) 


\section{REFERENCES}

1. W. K. Hayman, Multivalent functions, Cambridge Univ. Press, New York, 1958.

2. Z. J. Jakubowski, Sur les coefficients des fonctions univalentes dans le cercle unite, Ann. Polon. Math. 19 (1967), 207-233.

3. E. P. Merkes, M. S. Robertson and W. T. Scott, On products of starlike functions, Proc. Amer. Math. Soc. 6 (1962), 960-964.

4. H. S. Wilf, The radius of univalence of certain entire functions, Illinois J. Math. 6 (1962), 242-244.

UNIVERSITY OF KENTUCKY, LEXINGTON, KENTUCKY 40506 